\section{Experiência de cárie dentária na primeira dentição em município com fluoretação das águas}

\section{The prevalence of dental caries in milk teeth in a municipality with fluorinated water}

\section{Abstract}

Objectives: to determine the prevalence and severity of dental caries and the need to treat preschool aged between five and six years in a municipality with fluorinated water.

Methods: a cross-sectional study was carried out using a census examining 432 children aged between five and six years, of both sexes, from municipal public schools in Passo Fundo, in the Brazilian State of Rio Grande do Sul. An epidemiological survey was conducted using clinical examinations and demographic data on the pre-school children, in accordance with the methodology proposed by the World Health Organization. The investigators were duly trained in the application of common standards, obtaining a result of 0.91 on the Kappa test. The study was sent to and approved by the Research Ethics Committee of the Institute of Cardiology in Porto Alegre-RS.

Results: the ceo-d index was 4.1 ( \pm 3.73$)$ and only 106 (24.5\%) of children were free of caries in milk teeth; the prevalence of the need for treatment of caries was $69.9 \%$

Conclusions: a high prevalence of dental caries and widespread need for treatment was found, indicating that the oral health promotion strategies used in the municipality are still not proving to be effective.

Key words Prevalence, Dental caries, Epidemiology, Dentition, primary
Lilian Rigo 1

Eliane Alvim de Souza 2

Arnaldo de França Caldas Junior 3

1-3 Faculdade de Odontologia. Universidade de Pernambuco. Av Gal. Newton Cavalcanti, 1650. Tabatinga. Camaragibe, PE, Brasil. CEP: 54.753-220. E-mail: lilianrigo@via-rs.net

\section{Resumo}

Objetivos: determinar a prevalência e gravidade da cárie dentária e a necessidade de tratamento em pré-escolares de cinco e seis anos de idade em um município com fluoretação de águas.

Métodos: o desenho do estudo foi do tipo transversal caracterizado por um censo, sendo examinadas 432 crianças de cinco e seis anos, de ambos os sexos, das escolas da rede municipal de Passo Fundo, Rio Grande do Sul. O levantamento epidemiológico foi realizado por meio de exames clínicos e dados relativos às questões demográficas referentes aos préescolares, segundo metodologia proposta pela Organização Mundial da Saúde. Os examinadores foram devidamente treinados e calibrados, obtendo resultado do teste Kappa de 0,91. A pesquisa foi encaminhada e aprovada pelo Comitê de Ética em Pesquisa do Instituto de Cardiologia de Porto Alegre$R S$.

Resultados: o indice ceo-d foi de 4,1 $( \pm 3,73) e$ somente 106 (24,5\%) das crianças estavam livres de cárie dentária na dentição decídua; a prevalência da necessidade de tratamento de cárie foi de 69,9\%.

Conclusões: alta prevalência de cárie dentária e grande necessidade de tratamento foram encontradas, denotando que as estratégias de promoção de saúde bucal do município ainda não estão sendo efetivas.

Palavras-chave Prevalência, Cáries dentárias, Epidemiologia, Dentição primária 


\section{Introdução}

A partir dos anos 70 houve um expressivo declínio na prevalência da cárie dentária em diversos países desenvolvidos e em desenvolvimento, particularmente devido à larga utilização dos dentifrícios fluoretados e a aplicação de fluoretos nas águas de abastecimento público em inúmeras cidades.1-4 Paralelamente, a crescente utilização de dentifrícios fluoretados e a implantação de programas preventivos (conscientização da população em relação à saúde bucal e ênfase à educação para a saúde) contribuiu para a redução da prevalência de cárie no país. ${ }^{5}$ Narvai et al., ${ }^{6}$ analisando o declínio da cárie dentária no país no final do século XX, concluem que a descentralização do sistema de saúde foi um dos fatores que contribuíram igualmente para a evolução satisfatória dos índices de cárie entre as crianças e os jovens brasileiros.

Apesar da melhoria dos indicadores de saúde bucal observado nas últimas décadas, a cárie dentária ainda permanece como um grave problema de saúde pública no Brasil.2,3,7,8 No país, a distribuição na ocorrência de cárie entre as regiões é desigual, observando-se pior situação nos Estados do Nordeste, quando comparados aos Estados do Sul e Sudeste. 9

A Organização Mundial da Saúde (OMS), em 1981, e a Federação Dentária Internacional (FDI) estabeleceram como meta que pelo menos $50 \%$ das crianças entre cinco e seis anos de idade estivessem livres de cárie no ano 2000.10 No entanto, os resultados apresentados pelo último levantamento nacional, em 2003, SB Brasil - Levantamento das Condições de Saúde Bucal da População - mostraram que $40,6 \%$ de crianças estavam livres de cárie, percentual abaixo da meta estabelecida. ${ }^{9}$

A maior proporção de levantamentos de saúde bucal em crianças tem sido realizada em faixas etárias escolar - acima de seis anos de idade - em detrimento da faixa pré-escolar. 11 Provavelmente isso ocorre em razão da menor importância dada à dentição decídua ou pela dificuldade em realizar exames clínicos em crianças com menos de seis anos. A fim de evitar cáries dentárias e mutilações na dentição permanente, é de suma importância que os cuidados em saúde bucal sejam tomados desde a primeira dentição. ${ }^{12}$

Passo Fundo é um município de médio porte da Região Sul do país (185.279 habitantes), situado na região norte do Rio Grande do Sul, possui um Índice de Desenvolvimento Humano (IDH) de 0,80 e incorporação de flúor na água de abastecimento público desde 1975 (0,7 mg F/L). Em razão da ausência de estudos anteriores realizados na população escolar do município a realização de inquérito epidemiológico nessa população torna-se importante, pois possibilita a comparação dos resultados com os de levantamentos futuros e pode ser utilizado como referência para planejamento de estratégias de promoção de saúde bucal e indicador da qualidade dos serviços prestados em idades mais precoces.

O propósito do presente trabalho foi determinar a prevalência e severidade da cárie dentária e a necessidade de tratamento em pré-escolares de cinco e seis anos de idade no referido município.

\section{Métodos}

O desenho do estudo é tipo transversal e caracterizou-se por um censo, sendo examinadas todas as crianças de cinco e seis anos de idade, de ambos os sexos, matriculadas nas 26 Escolas Municipais de Educação Infantil (antigas creches) e 26 Escolas Municipais de Ensino Fundamental de Passo Fundo, Estado do Rio Grande do Sul, que estavam presentes nas visitas feitas à escola para o exame intrabucal. $\mathrm{O}$ estudo seguiu as orientações do levantamento epidemiológico (SB 2000) realizado no Brasil. ${ }^{9}$

$\mathrm{O}$ estudo foi encaminhado e aprovado pelo Comitê de Ética e Pesquisa do Instituto de Cardiologia de Porto Alegre, Rio Grande do Sul, sob $n^{\circ}$. de processo 174/05. Obteve-se a autorização dos responsáveis pelas crianças para a realização dos exames por meio do consentimento livre e esclarecido, com as informações do que seria realizado. Os termos foram enviados para todos os pais ou responsáveis pelas crianças de cinco e seis anos matriculadas nas escolas municipais de Passo Fundo $(\mathrm{n}=527)$, mediante listagens fornecidas pelas instituições.

Foram realizados exames clínicos bucais nas crianças nas salas de aula, utilizando-se espelho bucal, sonda e uma lanterna de mão.

A prevalência de cárie foi verificada pelos critérios recomendados pela OMS. ${ }^{13}$ A gravidade da cárie de cada criança foi avaliada considerando o número de dentes decíduos cariados, com extração indicada e obturados (ceo-d). Para cada elemento dentário, foi indicada a necessidade de tratamento.

Previamente aos exames clínicos, os três cirurgiões-dentistas examinadores participaram de um treinamento para padronizar e nivelar a coerência do diagnóstico de cárie segundo os critérios da OMS. ${ }^{13}$ A fim de garantir a confiabilidade dos dados sobre cárie dentária, realizou-se uma padronização de diagnóstico com um grupo de 20 crianças de uma das escolas, selecionadas aleatoriamente. As crianças 
foram examinadas duas vezes, com intervalo de uma semana, utilizando os mesmos critérios de diagnóstico. A estatística kappa mostrou concordância ótima interexaminadores nos diagnósticos realizados em cada um dos exames $($ kappa $=0,91)$. Um estudo piloto foi realizado com 40 alunos selecionados a fim de testar a metodologia proposta neste trabalho.

Os dados foram digitados no programa Epi Data versão 3.1 e exportados para análise no programa estatístico SPSS 11.0 for Windows.

A análise estatística descritiva baseou-se em cálculo de proporções, medidas de tendência central e de diversidade para a variável dependente cárie dentária (níveis do índice ceo-d). O critério usado para verificar a gravidade da cárie foi adaptado da OMS, 10 seguindo a distribuição das frequências dos dados das variáveis. A ausência de cárie foi representada pelo ceo- $d=0$, moderada prevalência foi representada por ceo-d de 1 a 3 , e alta prevalência representada pelo índice com valores de 4 em diante. Para a prevalência da necessidade de tratamento foram avaliados os dentes cariados e sem tratamento. A análise inferencial foi obtida através do teste do qui-quadrado, utilizado para testar a hipótese de igualdade e equivalência entre as proporções da amostra de crianças. O nível de significância adotado para os testes estatísticos foi de 5\% em um intervalo de confiança de $95 \%$. Os dados demográficos obtidos foram estratificados segundo idade, sexo e grupo étnico, utilizando a classificação proposta pelo Instituto Brasileiro de Geografia e Estatística. A variável grupo étnico foi categorizada em dois grupos: brancos e não brancos. Os dados do tipo de escola foram estratificados em Escola de Educação Infantil e Escola de Ensino Fundamental.

\section{Resultados}

De um total de 527 crianças, 432 participaram do estudo, tendo uma perda amostral de 95 (18\%), que ocorreu devido à ausência na escola nos dias agendados para o exame ou por não portarem a autorização de seus responsáveis para a realização da pesquisa.

Do total de crianças examinadas, 106 (24,5\%) pré-escolares estavam livres de cárie dentária. Categorizados segundo os níveis moderado e alto do índice ceo-d, 109 (25,2\%) apresentaram um ceo-d entre 1 e 3 , e $217(50,2 \%)$ igual ou maior do que 4, totalizando $326(75,4 \%)$ crianças com experiência de cárie dentária na dentição decídua.

A média do índice de cárie nos decíduos (ceo-d), na idade de cinco e seis anos, foi igual a $4,07( \pm 3,73$ e mediana 4,00$)$, sendo $4,14( \pm 3,74)$ e $3,9( \pm 3,72)$ no sexo masculino e feminino, respectivamente. As médias do número de dentes decíduos anteriores e do número de dentes decíduos posteriores acometidos por cáries nos pré-escolares foram as seguintes: $0,87( \pm 1,55)$ nos 12 dentes anteriores e $3,25( \pm 2,80)$ nos 8 dentes posteriores.

Foi analisado isoladamente o componente cariado do índice ceo-d, que fornece a informação sobre a frequência de crianças com necessidade de tratamento odontológico, e verificou-se que a frequência de dentes decíduos cariados correspondeu a $69,9 \%$ (302), sendo $70,8 \%$ meninos (177) e $68,7 \%$ meninas (125). O percentual de componente obturado foi de $22,9 \%$ (99 crianças). Os meninos ( $n=66$; $26,4 \%$ ) apresentaram maior proporção de tratamento restaurador (obturado) do que as meninas $(n=33$; $18,1 \%$ ), porém, como visto anteriormente, com maior prevalência de cárie. A média do componente cariado e sem restauração do índice ceo-d foi de 3,35 $( \pm 3,45)$, do componente obturado e cariado, 0,08 $( \pm 0,32)$, do obturado e sem cárie, $0,57( \pm 1,36)$ e cariado com extração indicada, $0,09( \pm 0,55)$.

Os dentes mais acometidos pela experiência de cárie dentária na dentição decídua foram os segundos molares (dentes $75,85,55$ e 65 , com $56 \%$, $55 \%, 46 \%$ e $45 \%$, respectivamente), seguidos pelos primeiros molares inferiores (dentes 84 e 74, com $39 \%$ e $38 \%$ ). Depois desses, foram os dentes centrais superiores (dentes 51 e 61 com $35 \%$ e $34 \%$ ) e os primeiros molares superiores (dentes 64 e $54 \mathrm{com}$ valores de $23 \%$ e $20 \%$ ). A Figura 1 apresenta as frequências das principais necessidades de tratamento (restauração de uma ou mais superfícies, restauração mais tratamento pulpar e extração indicada) dos dentes decíduos apresentados na descrição como os de maior prevalência de cárie.

Verificou-se a relação entre a presença de cárie anterior e o desenvolvimento de lesão nos molares decíduos, observando-se que 148 (95,5\%) das crianças que apresentaram cárie nos dentes anteriores, também apresentaram cárie nos dentes posteriores. Estavam livres de cárie nos dentes anteriores e posteriores $108(39,0 \%)$, de acordo com a Tabela 1.

Não houve associação significativa entre os níveis de ceo-d e as variáveis sócio-demográficas $(p>0,05)$, embora se tenha observado uma tendência na variável idade dos pré-escolares. As crianças menores (cinco anos) tiveram frequências maiores de dentes decíduos livres de cárie $(p=0,10)$. A Tabela 2 apresenta a distribuição da cárie segundo as variáveis sócio-demográficas: idade, etnia, sexo e tipo de escola frequentada pelas crianças. 
Prevalência da necessidade de tratamentos (restauração, tratamento endodôntico + restauração e extração dentária) dos dentes decíduos mais acometidos por cárie dentária nos pré-escolares do município de Passo Fundo, Rio Grande do Sul, 2008.

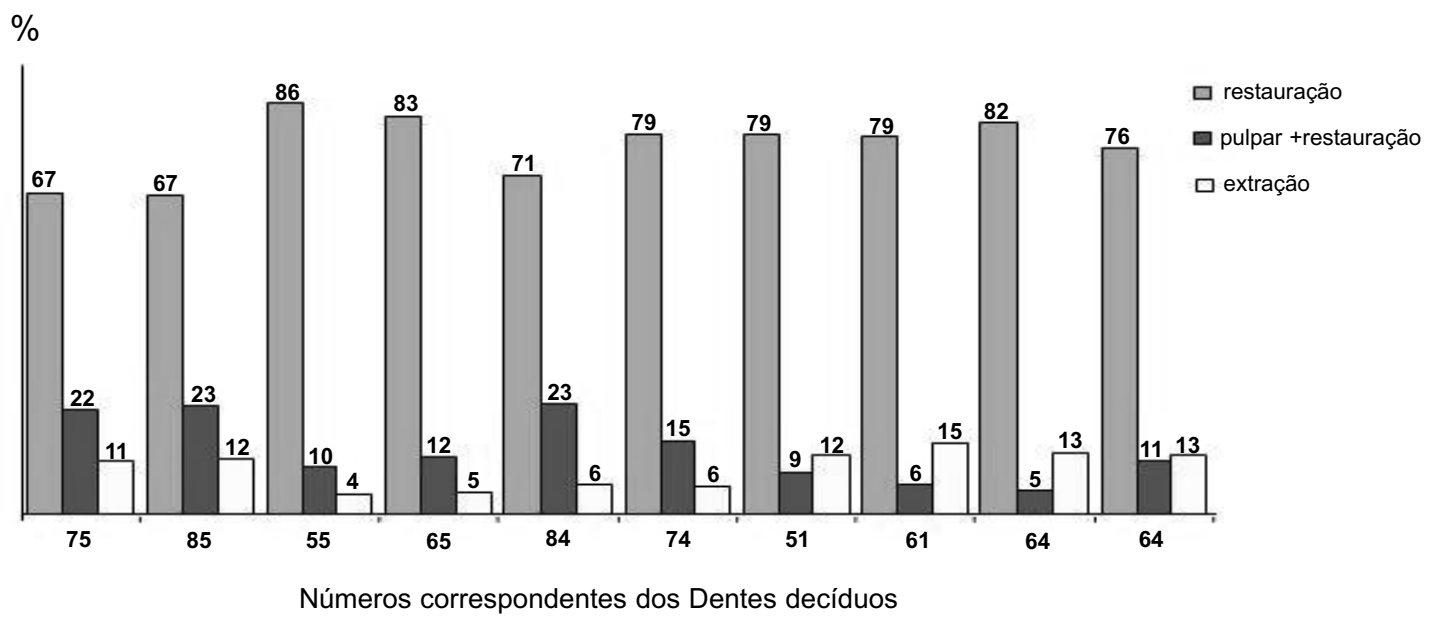

Tabela 1

Relação entre lesão de cárie nos dentes anteriores e nos dentes posteriores nos pré-escolares de Passo Fundo, Rio Grande do Sul, 2008.

\begin{tabular}{|c|c|c|c|c|c|c|c|}
\hline \multirow{3}{*}{ Lesão de cárie anterior } & \multicolumn{6}{|c|}{ Lesão de cárie posterior } & \multirow{3}{*}{$p^{*}$} \\
\hline & \multicolumn{2}{|c|}{ Não } & \multicolumn{2}{|c|}{ Sim } & \multicolumn{2}{|c|}{ Total } & \\
\hline & $\mathrm{n}$ & $\%$ & $\mathrm{n}$ & $\%$ & $\mathrm{n}$ & $\%$ & \\
\hline Não & & & & & & & $<0,001$ \\
\hline Sim & 7 & 4,5 & 148 & 95,5 & 155 & 100,0 & \\
\hline Total & 115 & 26,6 & 317 & 73,4 & 432 & 100,0 & \\
\hline
\end{tabular}

*Teste do qui-quadrado. 
Distribuição dos pré-escolares de acordo com os níveis do índice de cárie dentária (ceo-d) por idade, etnia, sexo e tipo de escola do município de Passo Fundo, Rio Grande do Sul, 2008.

\begin{tabular}{|c|c|c|c|c|c|c|c|c|c|}
\hline \multirow{3}{*}{ Variáveis } & \multicolumn{8}{|c|}{ Cárie dentária } & \multirow{3}{*}{$p^{*}$} \\
\hline & \multicolumn{2}{|c|}{ ceo-d 0} & \multicolumn{2}{|c|}{ ceo-d 1-3 } & \multicolumn{2}{|c|}{ ceo-d $>4$} & \multicolumn{2}{|c|}{ Total } & \\
\hline & $\mathrm{n}$ & $\%$ & $\mathrm{n}$ & $\%$ & $\mathrm{n}$ & $\%$ & $\mathrm{n}$ & $\%$ & \\
\hline \multicolumn{10}{|l|}{ Idade } \\
\hline 5 anos & 93 & 25,3 & 86 & 23,4 & 189 & 51,4 & 368 & 100,0 & 0,100 \\
\hline 6 anos & 13 & 20,3 & 23 & 35,9 & 28 & 43,8 & 64 & 100,0 & \\
\hline \multicolumn{10}{|l|}{ Etnia } \\
\hline Branco & 87 & 24,2 & 94 & 26,2 & 178 & 49,6 & 359 & 100,0 & 0,600 \\
\hline Não brancos & 19 & 26,0 & 15 & 20,5 & 39 & 53,4 & 73 & 100,0 & \\
\hline \multicolumn{10}{|l|}{ Sexo } \\
\hline Masculino & 61 & 24,4 & 65 & 26,0 & 124 & 49,6 & 250 & 100,0 & 0,909 \\
\hline Feminino & 45 & 24,7 & 44 & 24,2 & 93 & 51,1 & 182 & 100,0 & \\
\hline \multicolumn{10}{|l|}{ Tipo de escola } \\
\hline Escola Fundamental & 86 & 24,2 & 92 & 25,9 & 177 & 49,9 & 355 & 100,0 & 0,778 \\
\hline Escola Infantil & 20 & 26,0 & 17 & 22,1 & 40 & 51,9 & 77 & 100,0 & \\
\hline Total & 106 & 24,5 & 109 & 25,2 & 217 & 50,2 & 432 & 100,0 & \\
\hline
\end{tabular}

*Teste do qui-quadrado.

\section{Discussão}

O presente estudo avaliou a prevalência e a gravidade da cárie dentária e estimou a necessidade de tratamento dessa condição em 527 pré-escolares de cinco a seis anos de idade. O estudo foi caracterizado como um censo, contando com 81,97\% (432) da população alvo, portanto, representativa dessa população para as escolas de ensino da rede municipal da cidade de Passo Fundo.

Os resultados do presente estudo apontam uma alta prevalência de cárie dentária, e apenas $24,5 \%$ das crianças estavam livres de cárie dentária na dentição decídua. Constata-se, portanto, que a rede de escolas municipais ainda não atingiu a meta da OMS para o ano 2000. Esses achados corroboram com o resultado de estudo realizado em municípios rurais do Estado de São Paulo, nos anos de 1997 a 1999, em que apenas 19,55\% das crianças examinadas estavam livres de cárie. ${ }^{14}$ Em estudo realizado em 1996, verifica-se também que não houve melhora nos resultados, visto que $23,3 \%$ das crianças de cinco e seis anos estavam isentas de cárie na cidade de Bauru, porém, em São Paulo foram somente 9,3\%. 11 Aproximando-se um pouco mais da meta preconizada, um estudo em Piracicaba com pré-escolares municipais, obteve $44,3 \%$ de crianças livres de cárie aos cinco anos e $38,5 \%$ aos seis anos de idade. 15 No último levantamento nacional constatouse que a meta referente aos cinco anos, que estabelece uma proporção mínima de $50 \%$ de crianças livres de cáries, não foi atingida por nenhuma região do país. Entretanto, foram observadas diferenças entre as regiões, sendo a Sudeste a que mais se aproximou (44,92\% livre de cárie) e a Nordeste a mais distante $(34,92 \%) .{ }^{9}$ Contudo, estudo realizado em Recife, 16 cidade sem fluoretação das águas de abastecimento público, apresentou um percentual de $53 \%$ de livres de cárie e um ceo-d médio de 2,06, diferenciando-se dos escolares aqui estudados por terem a idade de quatro anos, inferior portanto, à faixa etária de cinco a seis anos, obtendo melhores resultados do que os do presente estudo. Isto pode ser atribuído aos grandes contrastes marcados pelas desigualdades sócio-demográficas entre as cidades das regiões brasileiras. Os indicadores de desenvolvimento social de uma localidade influenciam diretamente nas condições de saúde bucal da população.

A média do índice ceo-d verificada em Passo Fundo mostrou-se mais elevada do que em outros estudos relatados, incluindo estudo realizado em cidade de porte médio (Indaiatuba-SP), cuja média de dentes com experiência de cárie atingiu 1,73.17 
Com os resultados obtidos, observou-se que apesar de a cidade possuir água fluoretada há 32 anos, os índices de cárie dentária encontram-se em níveis nos quais ainda não atingiram aqueles encontrados em municípios onde possuem fluoretação das águas de abastecimento público há mais de 20 anos, assim como a cidade de Indaiatuba. O índice de cárie dos pré-escolares de cinco anos apresentou-se maior do que o encontrado em nível nacional (ceo-d=2,8) e também na Região Sul (ceo-d=2,62), ${ }^{9}$ sugerindo a implementação de procedimentos preventivos e curativos destinados a essa idade, uma vez que $69,9 \%$ das crianças com experiência de cárie necessitam de algum tipo de tratamento restaurador. Embora o município de Passo Fundo possua água fluoretada e um índice IDH elevado, as crianças investigadas frequentam escolas da rede pública e têm uma condição socioeconômica inferior, o que poderia levar a um resultado mais alto do índice ceo-d, quando comparado ao dos escolares da rede particular.

Embora haja escassez nos estudos relacionados à saúde bucal dos pré-escolares, a maioria dos trabalhos publicados relata prevalências expressivas da doença e das necessidades de tratamento odontológico.11,14,18-20 A grande necessidade de cuidado odontológico restaurador na dentição decídua pode ser traduzida como dificuldades do nível primário de atenção para executar as ações preventivas e curativas em saúde bucal na idade pré-escolar. 15 Além disso, é um desafio para os profissionais com relação ao controle do comportamento das crianças nessa faixa etária, o que também pode interferir na oferta de atenção odontológica para esse grupo. 16

O último levantamento epidemiológico nacional de 2003 confirma uma tendência à redução da prevalência da cárie aos 12 anos de idade, porém, nas crianças de zero a seis anos revela que a experiência de cárie na dentição decídua não mostrou redução nos índices, quando comparados a dados anteriores. ${ }^{9}$ Em média, uma criança de três anos no Brasil possui pelo menos um dente atacado por cárie e, aos cinco anos, pelo menos três, sendo o componente cariado do índice ceo-d o que compôs $80 \%$ do índice. ${ }^{9}$ As pesquisas sobre experiência de cárie dentária na primeira dentição mostram índices insatisfatórios, tanto em nível nacional como internacional. 19

Neste estudo verificou-se a necessidade de ampliar o acesso à atenção e o cuidado das famílias no desenvolvimento de hábitos para a saúde bucal desde a infância. O alto percentual de dentes decíduos cariados nessa faixa etária revela a necessidade de tratamento odontológico e sugere a pouca valo- rização por parte dos responsáveis e profissionais da saúde. ${ }^{20}$ Verifica-se na literatura, em relação a esses problemas, o descaso dos pais/responsáveis em relação à prevenção da doença na dentição decídua. ${ }^{21}$

No presente estudo, a prevalência de cárie foi mais alta nos dentes posteriores quando comparada aos dentes anteriores em ambos os sexos, sendo que os primeiros molares decíduos mostraram-se menos susceptíveis a cáries do que os segundos molares, embora estes erupcionem mais tarde. Semelhantes achados foram observados em outro estudo. 22 Explicações para esses resultados podem ser dadas, em razão da morfologia natural dos dentes molares. Os segundos molares decíduos apresentam sulcos e fissuras anatomicamente mais profundos na face oclusal do que os primeiros molares, favorecendo, por isso, um aumento da retenção de placa bacteriana e alimentos, elevando a prevalência de cárie, quando comparada aos primeiros molares. Adicionado a esses fatores, há uma grande dificuldade das crianças em higienizar os dentes posteriores.

Assim como relatado em outros estudos nacionais e internacionais, o sexo das crianças não se mostrou estatisticamente associado com a experiência de cárie dentária na dentição decídua; concluiu-se então que os grupos são homogêneos, apresentando distribuição igual da doença. 11,12,14,21,23 Por outro lado, achados recentes em área urbana de município da Índia com crianças de cinco anos, apresentaram prevalência de cárie mais alta nos meninos $(47,4 \%)$ do que nas meninas $(41,1 \%) .22$ Contudo, no presente estudo, quando se observou somente o componente obturado do índice ceo-d e o sexo das crianças, os meninos tiveram maior quantidade de dentes com tratamento restaurador do que as meninas, consequentemente menores necessidades de tratamento.

Não foram observadas diferenças estatisticamente significantes entre a idade das crianças e a ocorrência de cárie, ao contrário do observado em muitos estudos em que a idade é comumente um fator importante de influência na cárie dentária das crianças até cinco anos de idade. 21,23 Embora não tenha havido, no presente estudo, diferenças nas idades investigadas, as crianças mais velhas tenderam a apresentar maior frequência de cárie nos dentes decíduos, porque são esses os dentes há mais tempo na boca, consequentemente mais expostos aos fatores de risco à cárie. Uma explicação para os resultados do presente estudo não mostrarem associação pode ser atribuída à diferença de poucos meses em relação às duas idades estudadas. Seria necessário avaliar também os escolares de sete anos 
em diante para poder verificar com mais clareza se a prevalência da doença cárie na dentição decídua está aumentando com a idade.

Em relação ao tipo de escola, não foi possível estabelecer diferenças estatísticas entre Escolas Municipais de Educação Infantil (creches) e Escolas Municipais de Ensino Fundamental na relação com a experiência de cárie dentária. Estudos apresentados na literatura compararam escolas públicas com escolas privadas, verificando maior prevalência de cárie nas escolas da rede pública, observando ser uma variável sensível para discriminar diferentes condições de saúde bucal.8,24,25 Antunes et al.25 comentam a necessidade de avaliar as crianças aos cinco anos que já estão na escola e aquelas que ainda não ingressaram, pois supõe-se que as não frequentadoras de pré-escolas nessa idade possam apresentar condições socioeconômicas distintas das matriculadas nesses estabelecimentos. No presente estudo, houve preocupação de verificar se haviam diferenças quanto à doença nas crianças que frequentavam as Escolas de Educação Infantil, pois essas geralmente são de menor porte e onde as crianças permanecem durante os dois turnos. Já em relação às Escolas de Ensino Fundamental, são de maior porte, e, na maioria das vezes, as crianças ficam somente um turno por dia. Quanto aos hábitos da escola, observou-se que nas Escolas de Educação Infantil cada criança possuia uma escova dental na sala de aula e tinha o hábito da escovação diária, o que não ocorreu nas Escolas de Ensino Fundamental. Uma possível explicação por não ter encontrado diferenças significantes nesse estudo pode ser atribuído ao fato dos dois tipos de escolas pertencerem à rede municipal (mesmo tipo de alimentação) e situarem-se no mesmo bairro, apresentando condições socioeconômicas semelhantes.

Entre as limitações desse trabalho destacam-se o não acesso aos dados socioeconômicos. Contudo, com a utilização da variável etnia procurou-se verificar possíveis relações com a prevalência e a severidade com que as manifestações bucais ocorreram. Não foram encontradas associações estatísticas entre os grupos étnicos e cárie dentária, contrariando os achados do estudo de Antunes et al., ${ }^{26}$ no qual os autores observaram acesso desigual a tratamento odontológico entre crianças brancas e crianças negras e pardas, representando uma indicação adicional de disparidade sócio-demográfica afetando a experiência de cárie. No presente estudo verificouse que as crianças do município, independente do grupo étnico a que pertenciam, apresentaram alta prevalência de cárie e necessidades de tratamento odontológico.

Foi observada também alta ocorrência de cárie nos dentes anteriores das crianças que apresentavam cárie nos dentes posteriores decíduos. Esses achados evidenciaram associação e podem indicar ser preditor da doença em dentes molares decíduos. Corroborando com esses achados, outros estudos são importantes para essa discussão. Pereira et al. 27 relatam haver vários sinais diagnosticados como preditores de cárie nos dentes decíduos, porém destacam a relação da doença cárie nos dentes anteriores decíduos como preditora de cárie nos molares, com $73,1 \%$ das crianças estudadas apresentando lesão cariosa nos molares e nos dentes anteriores. Antunes et al. 25 observaram que a maioria das crianças que apresentavam cárie nos dentes anteriores possuía, também, nos dentes posteriores $(74,49 \%)$. Do mesmo modo, a maior parte das crianças livres da lesão nos dentes anteriores (90,79\%), estava igualmente livre de cárie nos molares decíduos. No estudo realizado por Ardenghi et al. 28 foi observada associação entre lesões de cárie na região anterior da maxila e na região de molares, em crianças de 12 a 59 meses de idade. Com o aumento da idade (a partir dos 24 meses) esta tendência foi maior. No presente estudo, verificou-se que as crianças com cárie em incisivos e caninos apresentaram uma forte tendência de desenvolvimento de lesões de cárie na região posterior, sendo necessária a implementação de medidas preventivas. É pertinente sugerir ao profissional, na abordagem odontológica das crianças na faixa etária entre 12 e 30 meses, atenção aos fatores clínicos de risco, observando criteriosamente as condições dos incisivos decíduos quanto à presença de placa visível, mancha branca de cárie, experiência pregressa de cárie e a erupção dos molares, a fim de introduzir precocemente ações preventivas que visem interromper o desenvolvimento de lesões detectadas ou impedir o surgimento de novas lesões. ${ }^{29}$

\section{Referências}

1. Cury JA. Uso do flúor. In: Baratieri LN et al. DentísticaProcedimentos preventivos e restauradores. São Paulo: Santos; 1989. p. 43-67.
2. Nadanowsky PA. O declínio da cárie. In: Pinto VG. Saúde Bucal Coletiva. 4 Ed. São Paulo: Santos; 2000. p. 341-51. 
3. Narvai PC. Cárie dentária e flúor: uma relação do século XX. Ciênc Saúde Coletiva. 2000; 5: 381-92.

4. Marinho VCC, Higgins JPT, Logan S, Sheian A. Fluoruru tópico (cremas dentales, enjuagues bucales, geles o barnices) para prevenir las caries dentales en niños y adolescentes. Em: La Biblioteca Cochrane Plus, 2007. Número 4. Oxford: update software 1td. [acesso em 2 jan 2007]. Disponível em: http://www.update/software.com.

5. Narvai PC. Está ocorrendo um declínio de cárie no Brasil? J ABOPREV. 1996; 7: 12

6. Narvai PC, Frazão P, Castellanos RA. Declínio na experiência de cárie em dentes permanentes de escolares brasileiros no final do século XX. Odontol Soc. 1999; 1 : 25-9.

7. Pinto VG. A odontologia no município: guia para organização de serviços e treinamento de profissionais a nível local. RGO (Porto Alegre). 1996; 253.

8. Traebert JL, Peres MA, Galesso ER, Zabot NE, Marcenes W. Prevalência e severidade da cárie dentária em escolares de seis a doze anos de idade. Rev Saúde Pública. 2001; 35 : 283-8.

9. Brasil. Ministério da Saúde. Secretaria de Políticas de Saúde. Departamento de Atenção Básica. Área Técnica de Saúde Bucal. Projeto SB2000: Condições de Saúde Bucal da População Brasileira no ano de 2000. Brasília, DF; 2004.

10. Fédération Dentaire Internacionale. Global goals for oral health in the year 2000. Int Dent J. 1982; 32: 1.

11. Tomita NE, Bijella VT, Lopes ES, Franco LJ. Prevalence of dental caries in preschool children attending nursery: the influence of socioeconomic factors. Rev Saúde Pública. 1996; 30: 413-20.

12. Scavuzzi ASF, Caldas Junior AF, Couto GBL, Vasconcelos MBBV, Soares RPF, Valença PAM. Longitudinal study of dental caries in Brazilian children aged from 12 to 30 months. Int J Paediatr Dent. 2007; 17: 123-8.

13. OMS (Organização Mundial da Saúde). Levantamentos básicos em saúde bucal. 4 Ed. São Paulo: Livraria Editora Santos; 1999.

14. Mello TRC, Antunes JLF, Waldman EA. Prevalência de cárie não tratada na dentição decídua em áreas urbanas e rurais do Estado de São Paulo. Rev Panam Salud Publica. 2008; 23: 78-84.

15. Cypriano S, Sousa MLR, Rihs LB, Wada RS. Saúde bucal de pré-escolares. Rev Saúde Pública. 2003; 37: 247-53.

16. Feitosa S, Colares V. Prevalência de cárie dentária em préescolares da rede pública de Recife, Pernambuco, Brasil, aos quatro anos de idade. Cad Saúde Pública. 2004; 20 : 604-9.

17. Rihs LB, Sousa MLR, Cypriano S, Abdalla NM, Guidini DDN, Amgarten C. Atividade de cárie na dentição decídua, Indaiatuba, São Paulo, Brasil, 2004. Cad. Saúde Pública. 2007; 23: 593-600.

Recebido em 19 de maio de 2008

Versão final apresentada em 25 de agosto de 2009

Aprovado em 30 de setembro de 2009
18. Abreu MHN, Pordeus IA, Modena CM. Cárie dentária entre escolares do meio rural de Itaúna (MG), Brasil. Rev Panam Salud Publica. 2004; 16: 334-44.

19. Pine CM, Adair PM, Petersen PE, Douglass C, Burnside G, Nicoll AD, Gillett A, Anderson R, Beighton D, Jin-You B, Broukal Z, Brown JP, Chestnutt IG, Declerck D, Devine D, Espelid I, Falcolini G, Ping FX, Freeman R, Gibbons D, Gugushe T, Harris R, Kirkham J, Lo ECM, Marsh P, Moupome G, Naidoo S, Ramos-Gomex F, Sutton BK, Williams S. Developing explanatory models of health inequalities in childhood dental caries. Community Dent Health. 2004; 21 : 86-95.

20. Batista, SPR. Análise dos principais indicadores em saúde bucal no Município de Campos dos Goytacazes/RJ, Brasil [Dissertação]. Piracicaba: Faculdade de Odontologia de Piracicaba da Universidade Estadual de Campinas; 2005.

21. Mahejabeen R, Sudha P, Kulkarni SS, Anegundi R. Dental caries prevalence among preschool children of Hubli: Dharwad city. J Indian Soc Pedod Prev Dent. 2006; 24: 1922.

22. Saravanan S, Madivanan I, Subashini B, Felix JW. Prevalence pattern of dental caries in the primary dentition among school children. Indian J Dent Res. 2005; 16: 140-6.

23. Namal N, Vehit HE, Can G. Risk factors for dental caries in Turkish preschool children. J Indian Soc Pedod Prev Dent. 2005; 23: 115-8.

24. Hoffmann RHS, Sousa MLR, Cypriano S, Souza R, Wada RS. Experiência de cárie dentária em crianças de escolas públicas e privadas de um município com água fluoretada. Cad Saúde Pública. 2004; 20: 435-44.

25. Antunes LAA, Antunes LS, Costa MEPR. Fatores utilizados como preditores de cárie na primeira Infância. Pesq Bras Odontoped Clin Integr. 2006; 6: 117-24.

26. Antunes JLF, Peres MP, Mello TRC. Determinantes individuais e contextuais da necessidade de tratamento odontológico na dentição decídua no Brasil. Cad Saúde Pública. 2006; 11: 79-87.

27. Pereira H P, Alves NCM, Riff GR, Magalhães EB, Costa, MEPR, Charlier, SC, Ferreira, FTSC, Neves, MLA. A doença cárie como preditor de cárie nos dentes decíduos e permanentes. Anais do SBPqO [online] 2003 [citado 25 jan 2008]. Disponível em: www.sbpqo.org.br/resumos/2003 /Issao_A.rtf

28. Ardenghi TM, Rodrigues CRMD, Bönecker M. Associação entre a prevalência de cárie em incisivos superiores e o desenvolvimento de cárie em molares decíduos. Anais do SBPqO [online] 2003 [citado 25 jan 2008]. Disponível em: http://www.sbpqo.org.br/resumos/2003/Painel_C_1.rtf.

29. Scavuzzi AIF, Oliveira VG, Ferreira EA. Incremento de Cárie Dental em Bebês. Pesq Bras Odontoped Clin Integr. 2007; 7: 161-7. 